Benchmarking Mean-Variance Portfolios Using a Shortage Function: The Choice of Direction Vector

Kristiaan Kerstens, Amine Mounir, Ignace Van de Woestyne

HUB RESEARCH PAPER 2010/1 JANUARI 2010

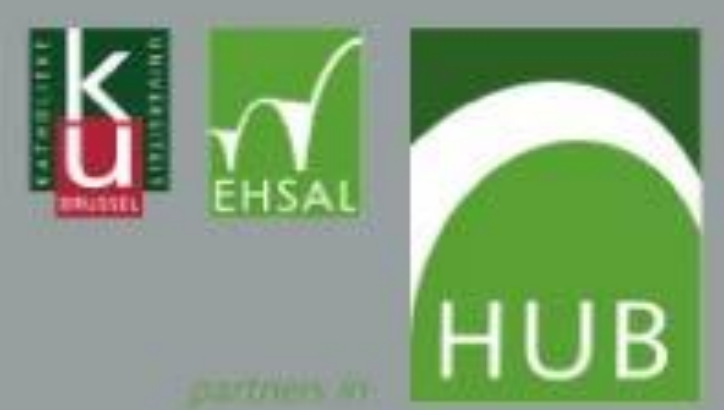




\title{
Benchmarking Mean-Variance Portfolios Using a Shortage Function: The Choice of Direction Vector
}

\author{
Kristiaan Kerstens, $\quad$ Amine Mounir† \\ Ignace Van de Woestyne
}

4th January 2010

\begin{abstract}
The shortage function has been proposed as a tool to gauge portfolio performance in multi-moment portfolio models. An open issue is how the choice of direction vector affects the efficiency measurement and, from a practical point of view, the resulting league tables. This paper illustrates empirically how the choice of direction vector affects the relative ranking of portfolios.
\end{abstract}

KEYWORDS: shortage function, efficient frontier, mean-variance efficiency

Warning: Please print the figures in colour for evaluation purposes

\section{Introduction}

Traditionally, the performance of portfolios within the mean-variance framework has been evaluated using performance measures which include some combination of information on both return and risk. Well-known classic examples are the Sharpe, Treynor and Jensen measures, among others (see Grinblatt and Titman (1989) for a critical discussion). Obviously, the menu of available portfolio performance measures has meanwhile been substantially expanded (see, e.g., Bacon (2008) or Feibel (2003) for surveys).

\footnotetext{
${ }^{*}$ CNRS-LEM (UMR 8179), IESEG School of Management, 3 rue de la Digue, F-59000 Lille, France, k.kerstens@ieseg.fr

${ }^{\dagger}$ Hogeschool Universiteit Brussel, Brussels, Belgium, amine.mounir@hubrussel.be

${ }^{\ddagger}$ Hogeschool Universiteit Brussel, Brussels, Belgium, ignace.vandewoestyne@hubrussel. be
} 
In production theory, the shortage function, that simultaneously looks for reductions in inputs and expansions in outputs and that is dual to the profit function, has been introduced by Luenberger (1995). ${ }^{1}$ Inspired by these developments in microeconomic theory where distance functions are used to characterise choice sets and to establish duality relations with value functions, Briec, Kerstens, and Lesourd (2004) introduced the shortage function as a portfolio performance gauging tool in the traditional mean-variance (MV) portfolio framework. In particular, these authors show that this shortage function can represent the MV-space and serves as an efficiency measure to position any portfolio with respect to the boundary of this portfolio set. Furthermore, developing a dual framework to assess the degree of satisfaction of investors preferences, they propose a decomposition of portfolio performance into allocative and portfolio efficiency. This approach was extended to the mean-variance-skewness (MVS) space in Briec, Kerstens, and Jokung (2007) where the shortage function seeks to project portfolios onto the MVS-frontier by looking for potential improvements (i.e., increasing return and skew, while decreasing risk). A framework for the general moment portfolio problem is described in Briec and Kerstens (2009), where the shortage function seeks to magnify odd moments and to decrease even moments.

This new shortage function approach has meanwhile been applied to hedge funds in a variety of contributions (see, e.g., Bacmann and Benedetti (2009), or Jurczenko and Yanou (2010)). It has also been contrasted to alternative models in Lozano and Guttiérez (2008), among others. Additional developments in this context are found in, e.g., Mencía and Sentana (2009).

Apart from portfolio theory, the utilisation of non-parametric frontier estimators in conjunction with the use of distance functions (that also have an efficiency interpretation) has found its way in a variety of finance topics. The performance of mutual funds has been rated along a multitude of dimensions (rather than mean and variance solely) using these boundary estimators. For instance, the seminal article of Murthi, Choi, and Desai (1997) employs return as a desirable output to be increased and risk and a series of transaction costs as an input to be reduced, and measures the performance of each mutual fund with respect to a piecewise linear frontier (rather than a traditional non-linear portfolio frontier). Most recently, employing a similar methodology, Glawischnig and Sommersguter-Reichmann (2010) critically compare the resulting higher order moment efficiency measures to traditional financial indices. In the context of asset selection and following the innovative article of Alam and Sickles (1998), similar ideas have been employed to show that changes in productive efficiency at least partially translate into changes in stock performance (see Edirisinghe and Zhang (2008) for a recent contribution).

All works (from Briec, Kerstens, and Lesourd (2004) to Briec and Kerstens (2009)) project any portfolio onto the portfolio frontier by using a direction vector directly related to the observed position of the portfolio itself. This choice of direction vector results in a proportional efficiency measure, which is convenient for practitioners. It is natural to ask how this choice of direction affects the efficiency

\footnotetext{
${ }^{1}$ Similar functions define duality relation in consumption theory (see Luenberger (1995)).
} 
assessment of portfolios.

Evident starting points to look for eventual readily available answers is the literature on the axiomatic foundations of efficiency measures in production theory and some further related literature in operations research. The analysis of the axiomatic foundations of efficiency measures in production theory goes back to at least Färe and Lovell (1978). Färe and Lovell (1978) initially proposed three axioms that an input-based efficiency index should satisfy: (i) indication (the index equals unity if and only if the input vector belongs to the strongly efficient subset), (ii) monotonicity (for constant other inputs and outputs, increasing an input must reduce the value of the index), and (iii) homogeneity of degree minus one (doubling inputs must halve the index). Later on, additional axioms have been proposed (e.g., Russell (1985, 1990) suggested (iv) invariance for units of measurement (commensurability) and ( $\mathrm{v}$ ) continuity in technology and also in input or output quantities). A recent article summarising this axiomatic literature is Russell and Schworm (2009a).

This literature can prove only partially inspiring, since it focused mainly on special distance functions that merely look for reduction in inputs (or improvements in outputs). However, the shortage function measures potential efficiency improvements in all dimensions. Russell and Schworm (2009b) recently took a look at similar efficiency measures in production theory and prudently conclude that the shortage function with a proportional interpretation satisfies a stronger unit invariance property compared to the case of a fixed direction. Furthermore, as may be clear from the above description so far, the traditional distance functions analysed in this axiomatic literature do not normally adhere to the mathematical notion of a norm (for exceptions, see Briec and Leleu (2003) for the use of a Hölder distance function). ${ }^{2}$ In brief, many alternatives to the proportional shortage function are conceivable, but this work remains silent on how to choose among the existing options.

Furthermore, a particular problem of portfolio theory compared to production theory is that returns and other odd moments can be negative, while production is normally confined to semi-positive input and output vectors. While in the nonparametric efficiency literature in production some articles do treat the case of negative numbers occurring in some particular specifications (e.g., growth in employment), this problem is omnipresent in a portfolio context. ${ }^{3}$ The work by, e.g., Silva Portela, Thanassoulis, and Simpson (2004) specifically deals with negative data when using a shortage function (see Pastor and Ruiz (2007) for a summary of these issues in the Data Envelopment Analysis (DEA) literature). As will be empirically documented, in portfolio analysis the traditional shortage function seems to lead to problems of performance measurement around the origin, a problem that hitherto went unnoticed in the literature.

Therefore, in this contribution we intend to systematically explore the conse-

\footnotetext{
${ }^{2}$ In finance, norms are used in a variety of contexts (see, e.g., the definition of coherent risk measures in Jarrow and Purnanandam (2005)), but -to the best of our knowledge- never to appraise portfolio performance.

${ }^{3}$ In operations research this literature is also known under the name Data Envelopment Analysis (DEA).
} 
quences of choosing different direction vectors for the shortage function in a MVportfolio context. In particular, this contribution is organised as follows. Section 2 provides some basic definitions as well as extensions in line with the framework developed in Briec, Kerstens, and Lesourd (2004). Section 3 offers a structured study of different choices of the direction vector in a basic Mean Variance (MV) context. In Section 4, making use of visualisations, the effect of these different choices is illustrated using an empirical example. The final Section 5 concludes and outlines potential future research avenues.

\section{Mean-Variance Portfolio Framework}

We start by briefly describing the non-parametric MV portfolio framework following the initial Briec, Kerstens, and Lesourd (2004) article, but extending and generalising it where appropriate.

Consider the basic problem of composing a portfolio from an investor's universe consisting of $n$ financial products, the latter referred to as the financial universe. A portfolio can then be represented by a weight vector $x=\left(x_{1}, \ldots, x_{n}\right)$. A sum constraint $\left(\sum_{i=1}^{n} x_{i}=1\right)$ determines the proportion of each of the initial products. Short selling is excluded, meaning that all weights $x_{i}$ are assumed to be positive. Consequently, the set of all portfolios, also known as the portfolio simplex, ${ }^{4}$ is the subset of $\mathbb{R}^{n}$ determined by

$$
\Im=\left\{x \in \mathbb{R}_{+}^{n} ; \sum_{i=1}^{n} x_{i}=1\right\} .
$$

The expected return vector and covariance matrix of the financial universe can be computed. More precisely, if $r_{i l}$ denotes the historical return of the $i^{\text {th }}$ financial product in the financial universe $(i=1, \ldots, n)$ at time $l(l=1, \ldots, m)$, then the expected return of the $i^{\text {th }}$ product over the given time window is equal to

$$
R_{i}=\frac{1}{m} \sum_{l=1}^{m} r_{i l}
$$

Furthermore, the covariance between the $i^{\text {th }}$ and the $j^{\text {th }}$ product, denoted by $V_{i j}$, is obtained by

$$
V_{i j}=\frac{1}{m} \sum_{l=1}^{m}\left(r_{i l}-R_{i}\right)\left(r_{j l}-R_{j}\right) .
$$

The expected return $\mathrm{E}[R(x)]$ of a portfolio $x$ and its variance $\operatorname{Var}[R(x)]$ can be

\footnotetext{
${ }^{4}$ This set of admissible portfolios can be modified to include additional constraints that can be written as linear functions of asset weights (e.g., transaction costs): see Briec, Kerstens, and Lesourd (2004). Briec and Kerstens (2009) also consider the cases of a risk-free asset and shorting.
} 
calculated as follows:

$$
\mathrm{E}[R(x)]=\sum_{i=1}^{n} x_{i} R_{i}, \quad \operatorname{Var}[R(x)]=\sum_{i, j=1}^{n} x_{i} x_{j} V_{i j} .
$$

The portfolio simplex $\Im$ is mapped into the 2-dimensional MV-space by means of the function $\Phi: \Im \rightarrow \mathbb{R}^{2}: x \mapsto \Phi(x)=(E[R(x)]$, $\operatorname{Var}[R(x)])$. The image set $\Phi(\Im)$ can be extended to the disposal representation set $\mathcal{D} \mathcal{R}=\Phi(\Im)+\left(\mathbb{R}_{-} \times \mathbb{R}_{+}\right)$.

Furthermore, every element of the 2-dimensional MV-space $\mathbb{R}^{2}$ is called a $\mathrm{MV}$ point. A MV-point can thus represent an observed portfolio in the sense that it can be the image by $\Phi$ of this portfolio, or can be an arbitrary point in this 2-dimensional space not related to a real portfolio. We also point out that both $\Phi(\Im)$ and $\mathcal{D} \mathcal{R}$ are contained in $\mathbb{R} \times \mathbb{R}_{+}$since the variance is always positive. As a consequence, both sets are situated in the first and fourth quadrant of the traditional MV-representation. Figure 1 provides an illustration generated from a fictitious financial universe.

Figure 1: Illustration of the image set $\Phi(\Im)$ and the disposal representation set $\mathcal{D} \mathcal{R}$.

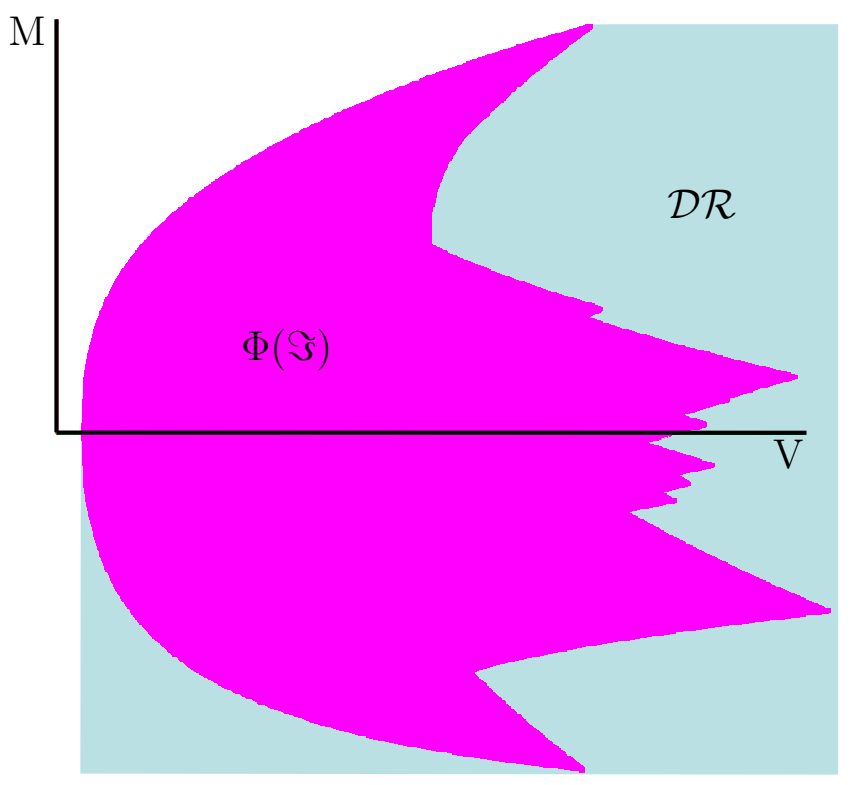

To introduce the notion of portfolio efficiency, the weakly efficient frontier is defined as a subset of $\mathcal{D} \mathcal{R}$ by:

Definition 2.1. In MV-space, the weakly efficient frontier is defined as:

$$
\partial^{W}(\mathcal{D} \mathcal{R})=\left\{\left(v_{M}, v_{V}\right) \in \mathcal{D} \mathcal{R} ;\left(-v_{M}^{\prime}, v_{V}^{\prime}\right)<\left(-v_{M}, v_{V}\right) \Rightarrow\left(v_{M}^{\prime}, v_{V}^{\prime}\right) \notin \mathcal{D} \mathcal{R}\right\} .
$$

Clearly, the weakly efficient frontier, also called the theoretical frontier, contains all $\mathrm{MV}$-points that are not weakly dominated in MV-space. Notice that by definition, 
this frontier is part of $\mathcal{D} \mathcal{R}$. As the latter is an extension of $\Phi(\Im)$, the theoretical frontier can also contain points not attainable by real portfolios.

The strongly efficient frontier is introduced as follows:

Definition 2.2. In MV-space, the strongly efficient frontier is defined as:

$$
\begin{aligned}
\partial^{S}(\mathcal{D} \mathcal{R})=\{ & \left(v_{M}, v_{V}\right) \in \mathcal{D} \mathcal{R} ;\left(-v_{M}^{\prime}, v_{V}^{\prime}\right) \leq\left(-v_{M}, v_{V}\right) \text { and } \\
& \left.\left(-v_{M}^{\prime}, v_{V}^{\prime}\right) \neq\left(-v_{M}, v_{V}\right) \Rightarrow\left(v_{M}^{\prime}, v_{V}^{\prime}\right) \notin \mathcal{D} \mathcal{R}\right\} .
\end{aligned}
$$

The strongly efficient frontier, shortened to efficient frontier, contains all MV-points that are not strictly dominated in MV-space. Now, the extended shortage function is introduced in the following definition:

Definition 2.3. Let $g=\left(g_{M}, g_{V}\right) \in \mathbb{R}_{+} \times \mathbb{R}_{-}$and $g \neq 0$. The extended shortage function $\mathcal{S}_{g}$ in the direction of vector $g$ is the function $\mathcal{S}_{g}: \mathbb{R}^{2} \rightarrow \mathbb{R}_{+} \cup\{-\infty\}$, with

$$
\mathcal{S}_{g}(v)=\sup _{\delta \in \mathbb{R}_{+}}\{\delta ; v+\delta g \in \mathcal{D} \mathcal{R}\} .
$$

The optimal value of $\delta$, if finite, is denoted by $\delta^{*}$. The corresponding vector $v^{*}=$ $v+\delta^{*} g$ is called the theoretical projected point.

Note firstly that the value $-\infty$ is only realised if the set $\{\delta ; v+\delta g \in \mathcal{D} \mathcal{R}\}$ is empty since it is commonly accepted that $\sup \varnothing=-\infty$. Secondly, the theoretical projected point $v^{*}$ is located at the weakly efficient frontier $\partial^{W}(\mathcal{D} \mathcal{R})$. Lastly, the extended shortage function (hereafter merely addressed as shortage function) extends the one introduced by Briec, Kerstens, and Lesourd (2004) and denoted by $\mathrm{S}_{g}$. The following diagram shows the relation between both functions:

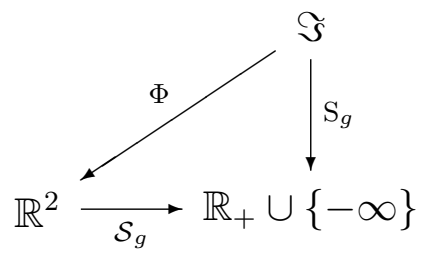

For a given direction vector $g=\left(g_{M}, g_{V}\right)$ specified as in Definition 2.3, the shortage function value for a MV-point $v=\left(v_{M}, v_{V}\right)$ under evaluation can be computed by solving the following quadratic non-linear model:

$$
\begin{array}{lrl}
\max _{x, \delta} & \delta & \\
\text { s.t. } \quad \sum_{i=1}^{n} x_{i} & =1, \\
\mathrm{E}[R(x)] & \geq v_{M}+\delta g_{M}, \\
\operatorname{Var}[R(x)] & \leq v_{V}+\delta g_{V}, \\
\delta \geq 0,0 \leq x_{i} & \leq 1 \text { for } i \in\{1, \ldots, n\} .
\end{array}
$$

As for the shortage function value, the following proposition is valid. 
Proposition 2.1. Let $v=\left(v_{M}, v_{V}\right) \in \mathcal{D} \mathcal{R}$, with $v \neq 0$, and $g$ the direction vector specified in Definition 2.3. Then $\mathcal{S}_{g}(v) \geq 0$, with $\mathcal{S}_{g}(v)=0 \Leftrightarrow v \in \partial^{W}(\mathcal{D} \mathcal{R})$.

Proof. Because the set $\{\delta ; v+\delta g \in \mathcal{D} \mathcal{R}\}$ is non-empty ( $\delta=0$ is a member), the result follows directly from Definition 2.3 of the shortage function.

From Proposition 2.1, we conclude that in practical shortage function value computations, three different cases can occur: (i) $\mathcal{S}_{g}(v)=-\infty$ if $v \notin \mathcal{D} \mathcal{R}$, which is observed as an infeasibility when solving model (P1); (ii) $\mathcal{S}_{g}(v)=0$ if $v$ is efficient; (iii) $\mathcal{S}_{g}(v)>0$ in all other cases, with higher values for less efficient vectors $v$. This observation makes the shortage function, for instance, suitable for ranking different financial products with respect to some financial universe: a higher shortage function value results in a lower ranking. ${ }^{5}$

Proposition 2.2. Let $g \in \mathbb{R}_{+} \times \mathbb{R}_{-}$be a non-zero direction vector. Then, $\mathcal{S}_{\lambda g}=\frac{1}{\lambda} \mathcal{S}_{g}$ for an arbitrary $\lambda>0$.

Proof. For an arbitrary vector $v$ in MV-space, it follows from Definition 2.3 that

$$
\mathcal{S}_{\lambda g}(v)=\sup _{\delta \in \mathbb{R}_{+}}\{\delta ; v+\delta \lambda g \in \mathcal{D} \mathcal{R}\}
$$

Now, let $\delta^{\prime}=\delta \lambda$. Then

$$
\mathcal{S}_{\lambda g}(v)=\sup _{\delta^{\prime} \in \mathbb{R}_{+}}\left\{\frac{\delta^{\prime}}{\lambda} ; v+\delta^{\prime} g \in \mathcal{D} \mathcal{R}\right\}=\frac{1}{\lambda} \cdot \sup _{\delta^{\prime} \in \mathbb{R}_{+}}\left\{\delta^{\prime} ; v+\delta^{\prime} g \in \mathcal{D} \mathcal{R}\right\}=\frac{1}{\lambda} \mathcal{S}_{g}(v) .
$$

Proposition 2.2 demonstrates the effect on the shortage function value of scaling the direction vector. A value $\lambda>1$ corresponds with an expansion of the direction vector, while $\lambda<1$ results in a contraction of this vector. Moreover, this proposition also shows that the computation of the shortage function value does not need to be redone after rescaling the direction vector. Indeed, the resulting value can be found directly by multiplying the initial shortage function values with the inverse of the scaling factor.

\section{Different Choices for the Direction Vector}

The shortage function provides a tool for measuring efficiency of a given portfolio with respect to the weakly efficient MV-frontier of some financial universe. Measuring is done in the direction of the vector $g$ with the purpose to simultaneously

\footnotetext{
${ }^{5}$ To obtain a positive relation between efficiency and the shortage function, one could consider taking the negative of the current definition. However, we prefer to stay in line with current practice in the literature.
} 
increase return and reduce risk. This explains the condition $g=\left(g_{M}, g_{V}\right) \in \mathbb{R}_{+} \times \mathbb{R}_{-}$ imposed in Definition 2.3. However, this restriction still leaves ample room for different choices. The literature so far has not described how to determine this direction vector and the impact of this choice on resulting computations (for instance, on efficiency based rankings of different portfolios).

Inspired by developments in economic theory, several choices can be proposed. ${ }^{6}$ One main distinction can be made between (i) a fixed direction, or (ii) a direction determined by the position of the portfolio under evaluation. Additionally, the direction vector could be rescaled or normed. In this section, we discuss each of these options in closer detail.

\subsection{Fixed Direction Vector}

A fixed direction vector in the shortage function definition is probably the most natural choice to start with. In this subsection, we investigate exactly this case.

Definition 3.1. The fixed direction vector, abbreviated as FD-vector, is a vector $g=$ $\left(c_{M},-c_{V}\right) \neq(0,0)$ with $c_{M}, c_{V} \geq 0$. Computations of the shortage function value (called FD-efficiency) and related vectors are done according to the FD-projection scheme if the FD-vector is used as direction vector.

By definition, the FD-vector points in an increasing return and decreasing risk direction if both $c_{M}$ and $c_{V}$ are distinct from zero. Figure 2 illustrates this type of FD-vector in the MV-plane.

Evidently, special directions can be considered (see, e.g., Morey and Morey (1999) for similar proposals). If $c_{M}=0$, then the FD-vector points in a horizontal direction in MV-space. This choice corresponds with a purely risk reducing strategy. The FD-vector points in a vertical upward direction in MV-space if $c_{V}=0$, corresponding with a purely return increasing strategy.

Apart from the horizontal and vertical directions, plenty of other directions can still be chosen in MV space. Commonly, one describes intermediate directions by means of an angle. For instance, an equal increase of return and decrease of risk strategy followed in Figure 2 leads to a $135^{\circ}$ angle.

Note that a correct interpretation of a given direction requires the same measuring unit on both axes of the MV figure. However, most frontier figures in MV-space, of which Figure 1 is an example, are scaled images, thereby optimising printing space and facilitating interpretation. More precisely, usually a different scaling is used on both axes leading to different units which evidently influences the angle of projection. Consequently, proposing a specific angle of direction should always be

\footnotetext{
${ }^{6}$ Briec (1997) introduces a position dependent direction vector in the efficiency literature. The article by, e.g., Chambers, Chung, and Färe (1998) opts for a fixed direction vector. Also Blackorby and Donaldson (1980) choose a fixed direction vector with unit coordinates when developing absolute inequality measures.
} 
Figure 2: Illustration of the FD-vector pointing to a risk reducing and return increasing direction.

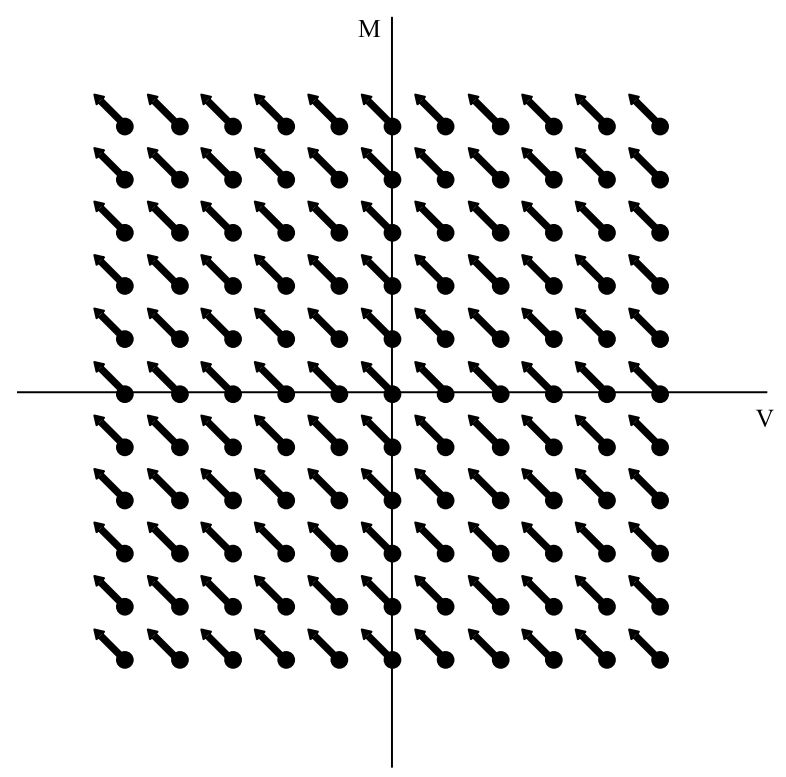

accompanied by information on the measuring units to allow for a correct interpretation.

As for the FD-efficiency, nothing can be added to the general Propositions 2.1 and 2.2. These propositions, however, guarantee that the shortage function can be used for ranking financial products on the basis of their efficiency with respect to the MV-frontier. Moreover, the ranking itself is not influenced by rescaling the FDvector. Only the exact direction of the direction vector needs to be determined. One way to go is to base the choice of direction on investor preferences (in particular, the choice of angle could reflect risk-aversion). ${ }^{7}$

\subsection{Unit Length Fixed Direction Vector}

The FD-vector $g$ in MV-space introduced in the previous subsection is taken rather arbitrarily. In particular, one could consider a vector with unit Euclidean length. ${ }^{8}$ This leads to the following definition:

Definition 3.2. The unit length fixed direction vector, abbreviated to UFD-vector, is a vector $g=\frac{1}{\sqrt{c_{M}^{2}+c_{V}^{2}}}\left(c_{M},-c_{V}\right) \neq(0,0)$ with $c_{M}, c_{V} \geq 0$. Computations of the shortage function value (called UFD-efficiency) and related vectors are done

\footnotetext{
${ }^{7}$ Briec, Kerstens, and Lesourd (2004) demonstrate that, due to dual relations between shortage function and mean-variance utility function, the shadow prices associated with the shortage function can yield information about investors risk aversion.

${ }^{8}$ Recall that the Euclidean length of a vector $v=\left(v_{M}, v_{V}\right)$ is given by $\sqrt{v_{M}^{2}+v_{V}^{2}}$. Note that instead of Euclidean length, other choices of norms could equally well be considered.
} 
according to the UFD-projection scheme if the UFD-vector is used as direction vector.

Clearly, the UFD-projection scheme is merely a special case of the FD-projection scheme, but with an adjusted direction vector. In fact, the following proposition clarifies the relationship between both projection schemes.

Proposition 3.1. Let $g_{F D}=\left(c_{M},-c_{V}\right)$ be a FD-direction vector. Then, the UFDefficiency equals the FD-efficiency multiplied by $\sqrt{c_{M}^{2}+c_{V}^{2}}$.

Proof. The result follows directly from Proposition 2.2 and the fact that the corresponding UFD-vector $g_{U F D}=\frac{1}{\sqrt{c_{M}^{2}+c_{V}^{2}}} g_{F D}$.

Notice that Figure 2 can also serve to illustrate the UPD-projection scheme. ${ }^{9}$

\subsection{Position Dependent Direction Vector}

Briec, Kerstens, and Lesourd (2004) opt for a direction vector $g$ depending on the position of the point to be mapped by the shortage function. More precisely, their choice is explained in the following definition.

Definition 3.3. Let $v=\left(v_{M}, v_{V}\right)$ be a point in MV-space. Then, the position dependent direction vector, abbreviated to $P D$-vector, is the vector $g=\left(\left|v_{M}\right|,-\left|v_{V}\right|\right)$. Computations of the shortage function value (called PD-efficiency) and related vectors are done according to the PD-projection scheme if the appropriate PD-vector is used as direction vector.

Clearly, the PD-vector points in a direction simultaneously increasing return and reducing risk. Moreover, this choice provides the shortage function with a convenient proportional interpretation, as can be seen from Proposition 3.2. ${ }^{10}$

Proposition 3.2. Let $v=\left(v_{M}, v_{V}\right) \in \mathcal{D} \mathcal{R}$, with $v \neq 0$, and $g$ the PD-vector. Then $0 \leq \mathcal{S}_{g}(v) \leq 1$, with $\mathcal{S}_{g}(v)=0 \Leftrightarrow v \in \partial^{W}(\mathcal{D} \mathcal{R})$.

Proof. Because of Proposition 2.1, we only need to prove additionally that $\mathcal{S}_{g}(v) \leq 1$ for $v=\left(v_{M}, v_{V}\right) \in \mathcal{D} \mathcal{R}$. Clearly, since $v_{V} \geq 0, g=\left(\left|v_{M}\right|,-v_{V}\right)$. Consequently,

$$
\mathcal{S}_{g}(v)=\sup _{\delta \in \mathbb{R}_{+}}\left\{\delta ;\left(v_{M}, v_{V}\right)+\delta\left(\left|v_{M}\right|,-v_{V}\right) \in \mathcal{D} \mathcal{R}\right\} .
$$

It now follows that $v_{V}(1-\delta) \geq 0$ which yields the required result.

\footnotetext{
${ }^{9}$ Normally, the arrows in the case of a fixed direction vector and a unit lenghth fixed direction vector would just differ in length. This would hardly be noticeable on a separate figure. To save space, we therefore refer to the same figure.

${ }^{10}$ As a matter of fact, as long as a portfolio model contains an even moment (variance, kurtosis, ... - i.e., all observed values in this dimension being stricly positive) this proprtional interpretation can be maintained.
} 
Figure 3 illustrates the PD-vector positioned at the corresponding point but without considering the proper length of the vector in an effort not to obscure the image. As can be observed, the PD-vector points towards the origin for original positions located in the fourth quadrant and points away from the origin for positions situated in the second quadrant. This phenomenon follows directly from the construction. Indeed, if $v=\left(v_{M}, v_{V}\right)$ is located in the fourth quadrant, then $v_{V} \geq 0$ and $v_{M} \leq 0$. Consequently, $g=\left(\left|v_{M}\right|,-\left|v_{V}\right|\right)=\left(-v_{M},-v_{V}\right)=-v$. Similarly, if $v$ is located in the second quadrant, then $v_{V} \leq 0$ and $v_{M} \geq 0$, resulting in $g=\left(\left|v_{M}\right|,-\left|v_{V}\right|\right)=\left(v_{M}, v_{V}\right)=v$. For points situated in the first or third quadrant, the resulting $\mathrm{PD}$-vector bends away from the origin.

Figure 3: Illustration of the PD-vector, only considering the direction and not the length of the vector, and positioned at the corresponding point.

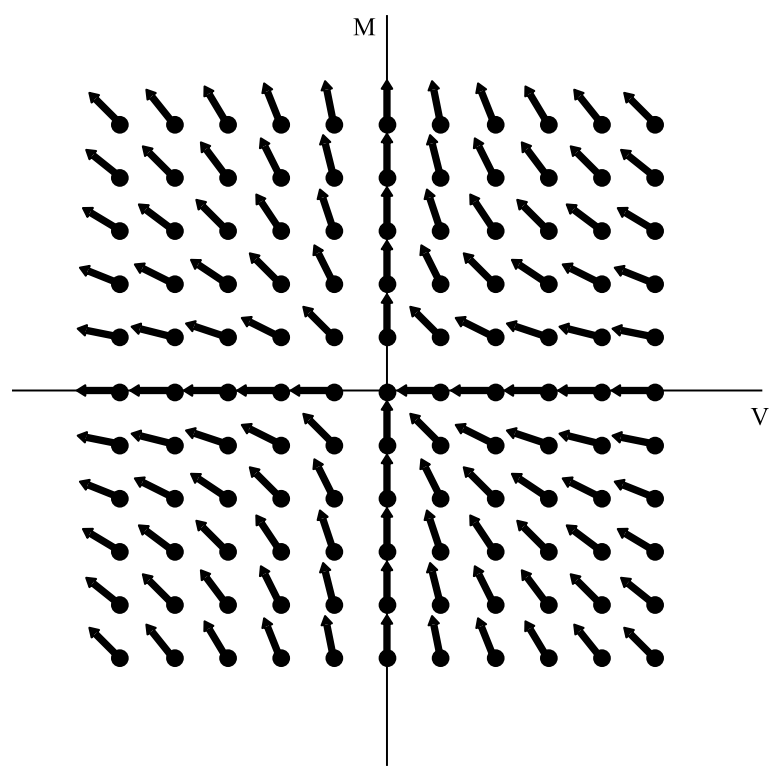

\subsection{Unit Length Position Dependent Direction Vector}

The PD-vector $g$ introduced in the previous subsection is completely determined by the position of the point $v$ in MV-space. In particular, the Euclidean length of the PD-vector varies when the position of $v$ changes. This could be undesirable. Therefore, in this subsection we examine the effect of switching to a direction vector that is still dependent on the position of $v$ but that has unit Euclidean length. We start by introducing the following definition:

Definition 3.4. Let $v=\left(v_{M}, v_{V}\right)$ be a point in MV-space distinct from the origin. Then, the unit length position dependent direction vector abbreviated to UPD-vector, is the vector $g=\frac{1}{\sqrt{v_{M}^{2}+v_{V}^{2}}}\left(\left|v_{M}\right|,-\left|v_{V}\right|\right)$. Computations of the shortage function value (called UPD-efficiency) and related vectors are done according to the $U P D$ projection scheme if the appropriate UPD-vector is used as direction vector. 
As is the case of the PD-vector, the UPD-vector points in a direction that increases return and decreases risk. In fact, Figure 3 can also perfectly serve as illustration of the UPD-projection scheme. However, the proportionality property (see Proposition 3.2) is no longer valid when choosing the UPD-vector. Instead, the following holds:

Proposition 3.3. Let $v \in \mathcal{D} \mathcal{R}$, with $v \neq 0$, and $g$ the UPD-vector. Then, $0 \leq$ $\mathcal{S}_{g}(v) \leq \sqrt{v_{M}^{2}+v_{V}^{2}} \cdot \mathcal{S}_{g}(v)=0 \Leftrightarrow v \in \partial^{W}(\mathcal{D} \mathcal{R})$.

Proof. The result follows directly from Propositions 2.2 and 3.2.

\section{Empirical Application}

Several projection schemes have been discussed in section 3 by means of their properties. In order to understand the differences more thoroughly, we apply these on a set of portfolios obtained from a real data base. Visualisations help in understanding the underlying patterns. Moreover, we explain the possible impact of different projection schemes on the efficiency based ranking of the portfolios under observation.

\subsection{Data Description}

The data base for this empirical part is obtained from Euronext and contains initially a selection of 101 assets (given by their daily returns from May 18 to October 1, 2009) traded at Euronext Paris. The assets are spread over Large Cap, Middle Cap, Small Cap, Free Market and Bonds. To obtain more detailed visualisations afterwards, this selection has been narrowed down to those assets with an expected return smaller than or equal to 0.006 and a variance less than or equal to 0.001 computed over the given time period. The financial universe on which we proceed thus contains 78 assets. $^{11}$

From the financial universe provided, twenty portfolios labelled from 1 to 20 are randomly drawn. Because of the financial universe's size, the portfolio weights are not reported here.

\subsection{Efficiency Computations and Discussion}

We start by visualising in Figure 4 the MV-frontier and the projection of the proposed portfolios onto this frontier according to different direction schemes. More precisely, we propose the PD- and UPD-projection schemes, and the FD- and UFDprojection schemes. The latter are computed in a horizontal, vertical and slant direction. As slant direction, we propose the FD-vector $g=\left(M_{\max },-V_{\max }\right)$, with

\footnotetext{
${ }^{11}$ Both the initial data base and the proposed selection are available upon simple request from the authors.
} 
$M_{\max }$ and $V_{\max }$ the maximal expected return and variance of all assets in the financial universe computed over the given time period, respectively. This choice of direction roughly corresponds, at least for this data base, with a projection in a $135^{\circ}$ direction when scaled to a square figure (see Figure $4 \mathrm{~b}$ ).

[Figure 4 about here]

The weakly efficient (green) and strongly efficient (red) MV-frontier, and the left side boundary of $\Phi(\Im)$ (blue) are visible on the left-hand side of each image of Figure 4. The original assets are visible as grey circles. The selected portfolios (black solid circles) are projected onto their optimal positions (black circles) on the MV-frontier according to the PD- and UPD-projection schemes in Figure 4a, and the FD- and UFD-projection schemes in Figures 4b (slant direction), 4c (horizontal direction), and $4 \mathrm{~d}$ (vertical direction). Notice that PD and UPD schemes lead to the same projected portfolios. The same remark applies to FD and UFD schemes. A detail of these images can be found in Figure 5. Notice that the scaling applied for the latter images visibly results in a different direction in Figure 5b compared with Figure 4b. This serves to illustrate what was mentioned earlier: providing a projection direction in terms of an angle should be accompanied by scaling information for correct interpretation.

[Figure 5 about here]

Next, the efficiencies of the twenty portfolios under observation are computed according to the proposed projection schemes. The resulting efficiencies are reported in Table 1.

[Table 1 about here]

Observe that the efficiencies computed according to FD- and UFD-projection schemes are equal up to a fixed factor. For example, the FD-efficiency in the slant direction equals the UFD-efficiency multiplied by 237.82 for all portfolios. Similar factors can be found for the other FD-directions. This result is a mere consequence of Proposition 3.1.

The noticeable differences in magnitude of different types of efficiencies make these mutually incomparable. It follows from Proposition 3.2 that the PD-efficiency can be interpreted as a proportional efficiency measure (or better inefficiency measure). Focussing on portfolio 10 for instance, the observed PD-efficiency of 0.883821 can be interpreted as an inefficiency of $88.38 \%$, knowing that all portfolios of the financial universe, and not only those under observation, have a PD-efficiency between $0 \%$ (fully efficient) and 100\% (fully inefficient). One might expect a similar interpretation for the FD-efficiency whose magnitude appears to be comparable with that of the PD-efficiency. Proposition 2.1, however, is the best one can say, meaning 
there is no predetermined upper bound for the FD-efficiencies. Consequently, the FD-efficiencies cannot be considered as proportional measures.

The UPD- and UFD-efficiencies are all pretty small. It follows from Definition 2.3 that, with these projection schemes, the shortage function value can be interpreted as the Euclidean distance to the MV-frontier in the appropriate direction. In fact, this interpretation makes it possible to compare the UPD- and UFD-efficiencies after all. Taking portfolio 10 again as an example, the slant UFD-efficiency is equal to 0.000322 , while the horizontal and the vertical UFD-efficiencies equal 0.000050 and 0.003050, respectively. Because of the shape of the MV-frontier (increasing and concave), one can expect that the slant UFD-efficiency is situated between the horizontal and the vertical UFD-efficiency. Also the UPD-efficiency of 0.000990 is situated between the horizontal and vertical UFD-efficiency. Clearly, this result is valid for all portfolios.

Based on the different efficiencies computed in Table 1, a ranking of all portfolios is established. The resulting rankings from best (smallest efficiency) to worst (largest efficiency) are listed in Table 2.

[Table 2 about here]

One is immediately struck by the differences between some of the rankings. Compare, for instance, the PD-ranking with the UPD-ranking. Portfolio 17, for example, performs best in the PD-ranking while its performance is rather average in the UPD-ranking. The differences can even be more pronounced. Portfolio 10, for instance, is the worst possible portfolio in the PD-ranking, which contrasts to its second best position in the UPD-ranking. Other examples show that this behaviour is not in any sense exceptional.

[Table 3 about here]

These casual observations are confirmed by Spearman's rank correlations represented in Table 3. Indeed, the rank correlation between the PD- and UPD-efficiencies is equal to 0.048 with a high significance of 0.840 . Therefore, both rankings can be considered statistically independent. Comparing other rankings, we come to the same conclusion except for the horizontal and the slant FD-UFD rankings. Here, we observe a statistically significant rank correlation of 0.986. Inspection of Table 2 confirms this result since one can notice that only the consecutive portfolios 14 and 17 are interchanged. Consequently, in this example, projecting horizontally or in the slant angle proposed earlier does not result in major differences.

[Figure 6 about here]

That projecting in different directions can lead to different rankings need not come as a surprise. Indeed, some portfolios can be situated close to the MV-frontier 
in one direction, but less close in other directions. The real surprise, however, is the drastic effect of switching from a PD-vector to the UPD-vector in the position dependent projection schemes, since in these cases, the directions are identical for both schemes.

To understand this unexpected behaviour, we visualise the different types of efficiencies in Figure 6. Starting from the financial universe, a particular rectangular region in MV-space covering $\Phi(\Im)$ is identified. On this region, a regular grid of size $200 \times 200$ is imposed. For each of the grid points $(40,000$ in total) the efficiency measure is computed, following one of the projection schemes. These values are then scaled to a grey tone value that can be used to colour the corresponding grid point. Consequently, the grey tone value gives an indication of the efficiency value. However, since the human mind is not well trained to discriminate between neighbouring grey tones, an additional transformation is used to cycle ten times through all grey tone values in consecutive increasing and decreasing order. Put differently, instead of using a pattern consisting of only one grey tone cycle (from black to white), a pattern consisting of ten cycles (from black to white and back to black) is utilised as colour function. As a consequence, different grey tone bands occur, all determined by MV-points with equal efficiency, thus, visualising the curves of equal efficiency. We mention that also the grid points are scaled to obtain square images.

Comparing Figures $6 \mathrm{a}$ and $6 \mathrm{~b}$, it becomes clear why switching to a unit length projection vector can have a major impact on the efficiency ranking. Indeed, the lines of equal UPD-efficiency seem to bend away around the origin, contrary to the lines of equal PD-efficiency. On the one hand, portfolios with an expected return of nearly zero and higher variance are promoted in the UPD-ranking compared with the PD-ranking. On the other hand, notice that the lines of equal PD-efficiency in Figure 6a are more condensed near the low variance side of the MV-frontier and close to the origin. This means that the PD-efficiency changes in this region more drastically than, for instance, in the high variance, high return area. Consequently, it is possible for portfolios to be situated rather close to the MV-frontier and still be considered as rather PD-inefficient. This effect should not be underestimated, especially in the presence of products with very low variance in the financial universe (which is quite often the case). As a consequence, portfolios in that area are almost always very inefficient, which is undesirable. The UPD-projection scheme tries to correct this effect, but actually overcompensates it as can be seen in Figure 6b.

When analysing Figures 6c, 6d and 6e, one notices that the lines of equal efficiency are translated in a horizontal, vertical, and slant direction, respectively. The resulting images correspond more closely to the idea that portfolios closer to the MV-frontier should have a higher efficiency ranking. Especially the slant FD- (and UFD-) projection scheme live up to this expectation. Remark that the horizontal FD- (and UFD-) projection concentrates the lines of equal efficiency near the high variance, high return area of the MV-frontier (see Figure 6c). A similar behaviour is noticeable in the low variance, low return area of the MV-frontier when projecting vertically according to a FD- (and UFD-) projection scheme (see Figure 6d). 


\section{Conclusions and Recommendations}

In section 3, we have described possible projection schemes for computing efficiency by means of the shortage function. From the empirical analysis in section 4, we have learned that different projection schemes can lead to different portfolio rankings. The PD-projection scheme has as advantage that its efficiency has a proportional interpretation (this is, having a value between zero and one), thereby making it suitable for situating the efficiency of any portfolio with respect to the financial universe. However, as a major drawback we observe that, in the presence of low variance products in the financial universe, portfolios with a low variance and low return have a rather high PD-efficiency. Therefore, these types of portfolios are conceived as being inefficient. Put differently, the PD-efficiency of high variance, high return products is less sensitive to changes compared to low variance, low return products. Consequently, high variance, high return products are usually promoted in PD-rankings.

The UPD-projection scheme tries to correct the drawback in the PD-projection scheme but actually overcompensates by bending the lines of equal UPD-efficiency away near the origin of the MV-plane. As a consequence, portfolios with a higher variance but a return close to zero are over-promoted in the UPD-ranking. This effect can equally lead to undesirable effects in the ranking. The UPD-efficiency has the advantage, however, that it can be interpreted as a true Euclidean distance to the MV-frontier in the appropriate direction. Therefore, its interpretation concurs with the natural idea of efficiency as some kind of distance to the frontier: smaller distances to the frontier result in smaller efficiency values.

The FD- and UFD-projection schemes lead to identical rankings, at least for the same fixed direction. When applying a UFD-projection scheme, the corresponding UFD-efficiency can be interpreted as a Euclidean distance to the MV-frontier measured in that direction. However, the FD- and UFD-efficiencies cannot be interpreted as proportional efficiency measures. The computation of one efficiency value can, therefore, not be interpreted, which should not be a problem when applied for ranking portfolios on the basis of their efficiency.

To summarise, all projection schemes considered here seem to have their specific advantages and drawbacks in a MV portfolio context. Therefore, an ideal projection scheme is not apparent at this moment. When it comes to efficiency based ranking of portfolios, however, we are in favour of the slant UFD-projection scheme since (a) the UFD-efficiency can be interpreted as a Euclidean distance to the MV-frontier, which is therefore interpretable (i.e., points closer to the frontier are more efficient); (b) the lines of equal UFD-efficiency in Figure 6e correspond most to our expectations (i.e., these basically have a similar shape as the original frontier).

This paper is, to the best of our knowledge, the first in systematically examining the effect of different projection schemes for the shortage function in a MV portfolio setting. Clearly, this research is not finished. It could, for instance, be interesting to look for the mathematical reasons why the lines of equal UPD-efficiency bend 
away near the origin of the MV-space. Furthermore, instead of using the Euclidean distance, other distance norms can be considered. The effect of other norm choices on the corresponding efficiencies and on related rankings is unknown so far. Finally, it could be equally interesting to examine the results of this analysis in production theory where the problem by its very nature is always multi-dimensional.

\section{References}

Alam, I., And R. Sickles (1998): "The Relationship Between Stock Market Returns and Technical Efficiency Innovations: Evidence from the US Airline Industry," Journal of Productivity Analysis, 9(1), 35-51.

Bacmann, J.-F., And S. Benedetti (2009): "Optimal Bayesian Portfolios of Hedge Funds," International Journal of Risk Assessment and Management, 11(12), 39-58.

Bacon, C. (2008): Practical Portfolio Performance: Measurement and Attribution. Wiley, New York, 2 edn.

Blackorby, C., and D. Donaldson (1980): "A Theoretical Treatment of Indices of Absolute Inequality," International Economic Review, 21(1), 107-136.

Briec, W . (1997): "A Graph-Type Extension of Farrell Technical Efficiency Measure," Journal of Productivity Analysis, 8(1), 95-110.

Briec, W., And K. Kerstens (2009): "Portfolio Selection in Multidimensional General and Partial Moment Space," Journal of Economic Dynamics and Control, forthcoming.

Briec, W., K. Kerstens, and O. Jokung (2007): "Mean-Variance-Skewness Portfolio Performance Gauging: A General Shortage Function and Dual Approach," Management Science, 53(1), 135-149.

Briec, W., K. Kerstens, and J. Lesourd (2004): "Single Period Markowitz Portfolio Selection, Performance Gauging and Duality: A Variation on the Luenberger Shortage Function," Journal of Optimization Theory and Applications, 120(1), 1-27.

Briec, W., ANd H. Leleu (2003): "Dual Representations of Non-Parametric Technologies and Measurement of Technical Efficiency," Journal of Productivity Analysis, 20(1), 71-96.

Chambers, R., Y. Chung, and R. Färe (1998): "Profit, Directional Distance Functions, and Nerlovian Efficiency," Journal of Optimization Theory and Applications, 98(2), 351-364.

Edirisinghe, N., And X. Zhang (2008): "Portfolio Selection under DEA-Based Relative Financial Strength Indicators: Case of US Industries," Journal of the Operational Research Society, 59(6), 842-856. 
Färe, R., AND C. Lovell (1978): "Measuring the Technical Efficiency of Production," Journal of Economic Theory, 19(1), 150-162.

FeiBel, B. (2003): Investment Performance Measurement. Wiley, New York.

Glawischnig, M., and M. Sommersguter-Reichmann (2010): "Assessing the Performance of Alternative Investments using Non-Parametric Efficiency Measurement Approaches: Is It Convincing?," Journal of Banking and Finance, 34(2), $295-303$.

Grinblatt, M., and S. Titman (1989): "Portfolio Performance Evaluation: Old Issues and New Insights," Review of Financial Studies, 2(3), 393-421.

Jarrow, R., And A. Purnanandam (2005): "A generalized coherent risk measure: The firm's perspective," Finance Research Letters, 2(1), 23-29.

Jurczenko, E., and G. Yanou (2010): "Fund of Hedge Funds Portfolio Selection: A Robust Non-parametric Multi-moment Approach," in Emerging Trends in Hedge Fund Strategies, ed. by Y. Watanabe, p. forthcoming. Nova Science, New York.

Lozano, S., and E. GuttiéRez (2008): "TSD-Consistent Performance Assessment of Mutual Funds," Journal of the Operational Research Society, 59(10), $1352-1362$.

Luenberger, D. (1995): Microeconomic Theory. McGraw-Hill, Boston.

Mencía, J., And E. Sentana (2009): "Multivariate Location-Scale Mixtures of Normals and Mean-Variance-Skewness Portfolio Allocation," Journal of Econometrics, 153(2), 105-121.

Morey, M., And R. Morey (1999): "Mutual Fund Performance Appraisals: A Multi-Horizon Perspective With Endogenous Benchmarking," Omega, 27(2), 241258.

Murthi, B., Y. Choi, and P. Desai (1997): "Efficiency of Mutual Funds and Portfolio Performance Measurement: A Non-Parametric Approach," European Journal of Operational Research, 98(2), 408-418.

PAstor, J., AND J. Ruiz (2007): "Variables with Negative Values in DEA," in Modeling Data Irregularities and Structural Complexities in Data Envelopment Analysis, ed. by J. Zhu, and W. Cook, pp. 63-84. Springer, Berling.

Russell, R. (1985): "Measures of Technical Efficiency," Journal of Economic Theory, 35(1), 109-126.

(1990): "Continuity of Measures of Technical Efficiency," Journal of Economic Theory, 51(2), 255-267.

Russell, R., And W. Schworm (2009a): "Axiomatic Foundations of Efficiency Measurement on Data-Generated Technologies," Journal of Productivity Analysis, $31(2), 77-86$. 
(2009b): "Axiomatic Foundations of Inefficiency Measurement on 〈input, output) Space," Journal of Productivity Analysis, p. forthcoming.

Silva Portela, M., E. Thanassoulis, and G. Simpson (2004): "Negative Data in DEA: A Directional Distance Approach Applied to Bank Branches," Journal of the Operational Research Society, 55(10), 1111-1121.

Table 1: Efficiencies of twenty randomly selected portfolios according to different projection schemes.

\begin{tabular}{rcccccccc}
\hline $\mathrm{Nr}$ & PD & UPD & FD hor & UFD hor & FD ver & UFD ver & FD slant & UFD slant \\
\hline 1 & 0.692152 & 0.001396 & 0.070869 & 0.000064 & 0.406139 & 0.002387 & 0.096315 & 0.000405 \\
2 & 0.779754 & 0.001156 & 0.046033 & 0.000042 & 0.436797 & 0.002567 & 0.063938 & 0.000269 \\
3 & 0.443501 & 0.001183 & 0.058670 & 0.000053 & 0.281548 & 0.001655 & 0.077089 & 0.000324 \\
4 & 0.708772 & 0.001183 & 0.041753 & 0.000038 & 0.394698 & 0.002320 & 0.057667 & 0.000242 \\
5 & 0.591681 & 0.001388 & 0.074082 & 0.000067 & 0.359669 & 0.002114 & 0.099206 & 0.000417 \\
6 & 0.811610 & 0.001549 & 0.125345 & 0.000114 & 0.494799 & 0.002908 & 0.170012 & 0.000715 \\
7 & 0.679392 & 0.001221 & 0.044759 & 0.000041 & 0.382507 & 0.002248 & 0.061511 & 0.000259 \\
8 & 0.794476 & 0.001287 & 0.064905 & 0.000059 & 0.458695 & 0.002696 & 0.089582 & 0.000377 \\
9 & 0.694604 & 0.001519 & 0.098996 & 0.000090 & 0.420354 & 0.002470 & 0.133121 & 0.000560 \\
10 & 0.883821 & 0.000990 & 0.054667 & 0.000050 & 0.519052 & 0.003050 & 0.076607 & 0.000322 \\
11 & 0.836747 & 0.001369 & 0.091765 & 0.000083 & 0.501430 & 0.002947 & 0.126227 & 0.000531 \\
12 & 0.526507 & 0.001357 & 0.081001 & 0.000074 & 0.334255 & 0.001964 & 0.106652 & 0.000448 \\
13 & 0.756302 & 0.001491 & 0.095947 & 0.000087 & 0.451748 & 0.002655 & 0.130262 & 0.000548 \\
14 & 0.736745 & 0.001550 & 0.109030 & 0.000099 & 0.445507 & 0.002618 & 0.146967 & 0.000618 \\
15 & 0.789060 & 0.000979 & 0.031841 & 0.000029 & 0.431482 & 0.002536 & 0.044565 & 0.000187 \\
16 & 0.883182 & 0.001631 & 0.207546 & 0.000188 & 0.566834 & 0.003331 & 0.278942 & 0.001173 \\
17 & 0.443159 & 0.001339 & 0.114992 & 0.000104 & 0.305361 & 0.001795 & 0.143802 & 0.000605 \\
18 & 0.873161 & 0.001113 & 0.065143 & 0.000059 & 0.516351 & 0.003034 & 0.090873 & 0.000382 \\
19 & 0.658802 & 0.001509 & 0.098974 & 0.000090 & 0.403417 & 0.002371 & 0.132366 & 0.000557 \\
20 & 0.873004 & 0.001098 & 0.063138 & 0.000057 & 0.514912 & 0.003026 & 0.088129 & 0.000371 \\
\hline
\end{tabular}


Table 2: Ranking of twenty randomly selected portfolios according to different types of efficiency.

\begin{tabular}{crrrcc}
\hline Ranked by & PD & UPD & FD-UFD hor & FD-UFD ver & FD-UFD slant \\
\hline best & 17 & 15 & 15 & 3 & 15 \\
& 3 & 10 & 4 & 17 & 4 \\
& 12 & 20 & 7 & 12 & 7 \\
& 5 & 18 & 2 & 5 & 2 \\
& 19 & 2 & 10 & 7 & 10 \\
& 7 & 4 & 3 & 4 & 3 \\
& 1 & 3 & 20 & 19 & 20 \\
& 9 & 7 & 8 & 1 & 8 \\
& 4 & 8 & 18 & 9 & 18 \\
& 14 & 17 & 1 & 15 & 1 \\
& 13 & 12 & 5 & 2 & 5 \\
& 2 & 11 & 12 & 14 & 12 \\
& 15 & 5 & 11 & 13 & 11 \\
& 8 & 1 & 13 & 8 & 13 \\
& 6 & 13 & 19 & 6 & 19 \\
& 11 & 19 & 9 & 11 & 9 \\
\hline \multirow{5yyyy}{*}{ worst } & 20 & 9 & 14 & 20 & 17 \\
\hline & 18 & 6 & 17 & 18 & 14 \\
& 16 & 14 & 6 & 10 & 6 \\
\hline
\end{tabular}

Table 3: Spearman's Rank Correlation between Different Efficiency Rankings

\begin{tabular}{|rr|r|r|r|r|c|}
\hline & & PD & UPD & $\begin{array}{c}\text { FD-UFD } \\
\text { hor }\end{array}$ & $\begin{array}{c}\text { FD-UFD } \\
\text { ver }\end{array}$ & $\begin{array}{c}\text { FD-UFD } \\
\text { slant }\end{array}$ \\
\hline PD & Rank Correlation & 1.000 & 0.048 & -0.063 & 0.023 & -0.054 \\
& Sig. (2-tailed) & & 0.840 & 0.791 & 0.925 & 0.821 \\
\hline UPD & Rank Correlation & 0.048 & 1.000 & -0.314 & -0.026 & -0.301 \\
& Sig. (2-tailed) & 0.840 & & 0.177 & 0.915 & 0.198 \\
\hline FD-UFD hor & Rank Correlation & -0.063 & -0.314 & 1.000 & 0.284 & $0.986^{* *}$ \\
& Sig. (2-tailed) & 0.791 & 0.177 & & 0.225 & 0.000 \\
\hline FD-UFD ver & Rank Correlation & 0.023 & -0.026 & 0.284 & 1.000 & 0.293 \\
& Sig. (2-tailed) & 0.925 & 0.915 & 0.225 & & 0.210 \\
\hline FD-UFD slant & Rank Correlation & -0.054 & -0.301 & $0.986^{* *}$ & 0.293 & 1.000 \\
& Sig. (2-tailed) & 0.821 & 0.198 & 0.000 & 0.210 & \\
\hline
\end{tabular}

**: Correlation is significant at the 0.01 level (2-tailed). 
Figure 4: Projection of selected portfolios onto the MV-frontier following different projection schemes.

Visualisation of the weakly efficient (green) and strongly efficient (red) MV-frontier, and the left side boundary of $\Phi(\Im)$ (blue). The original assets are visible as grey circles. The selected portfolios (black solid circles) are projected onto their optimal positions (black circles) on the MV-frontier according to the following projection schemes: (a) The PD- and UPD-projection scheme; (b) The FD- and UFDprojection scheme in a slant direction; (c) The FD- and UFD-projection scheme in a horizontal direction; (d) The FD- and UFD-projection scheme in a vertical direction.

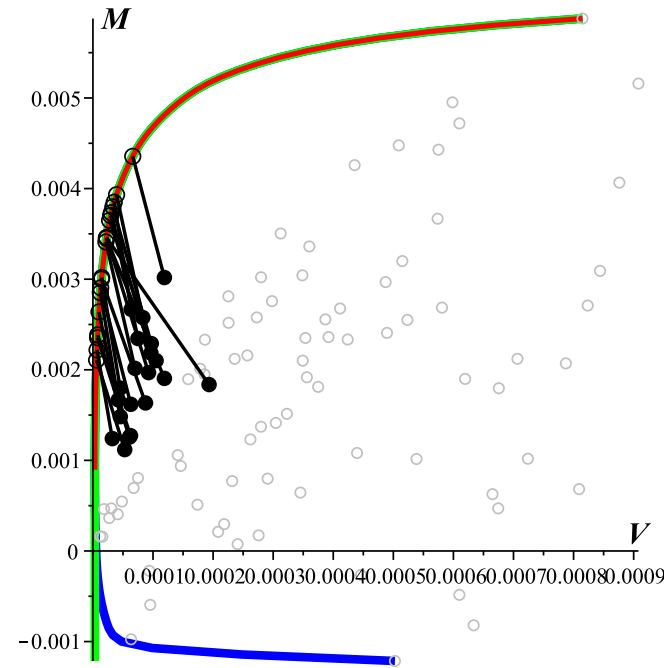

(a)

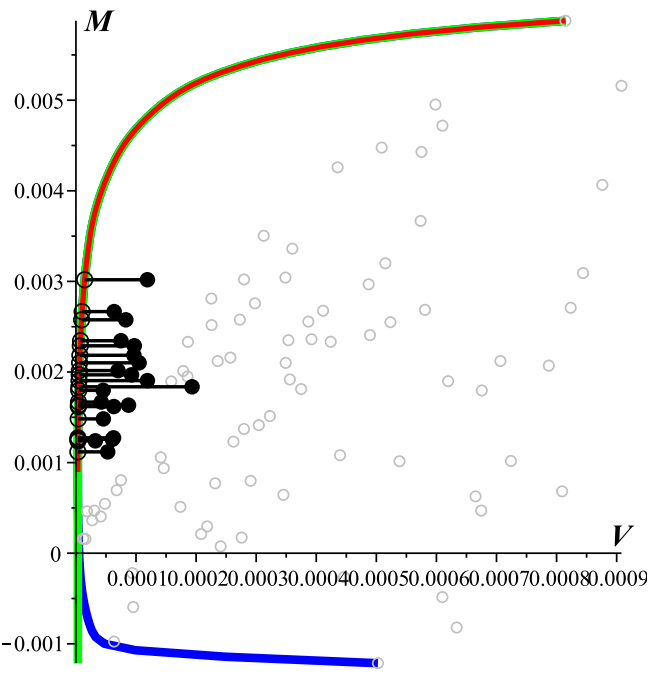

(c)

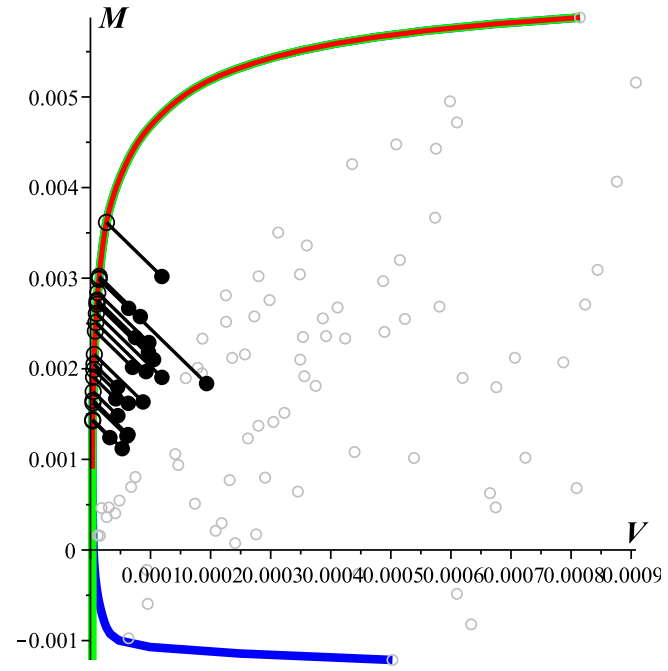

(b)

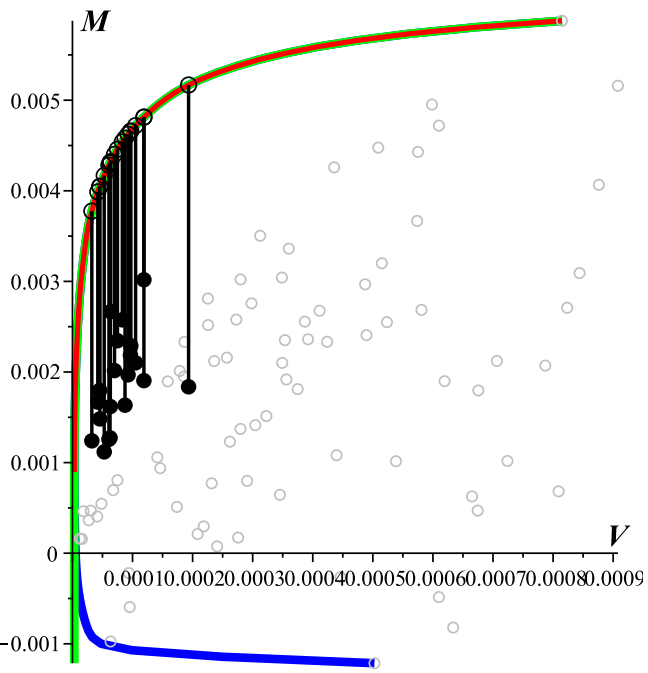

(d) 
Figure 5: Details of the projection of selected portfolios onto the MVfrontier following different projection schemes.

Visualisation of the weakly efficient (green) and strongly efficient (red) MV-frontier. Some of the original assets are visible as grey circles. The selected portfolios (black solid circles) are projected onto their optimal positions (black circles) on the MVfrontier according to the following projection schemes: (a) The PD- and UPDprojection scheme; (b) The FD- and UFD-projection scheme in a slant direction; (c) The FD- and UFD-projection scheme in a horizontal direction; (d) The FD- and UFD-projection scheme in a vertical direction.

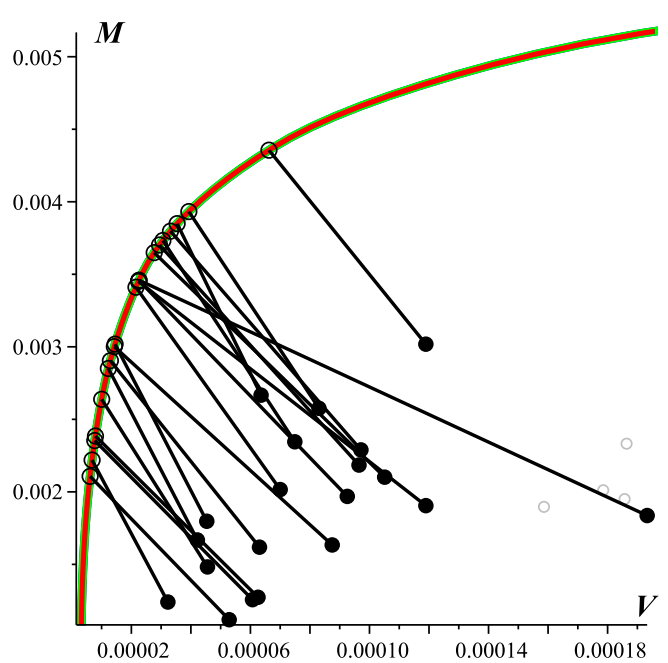

(a)

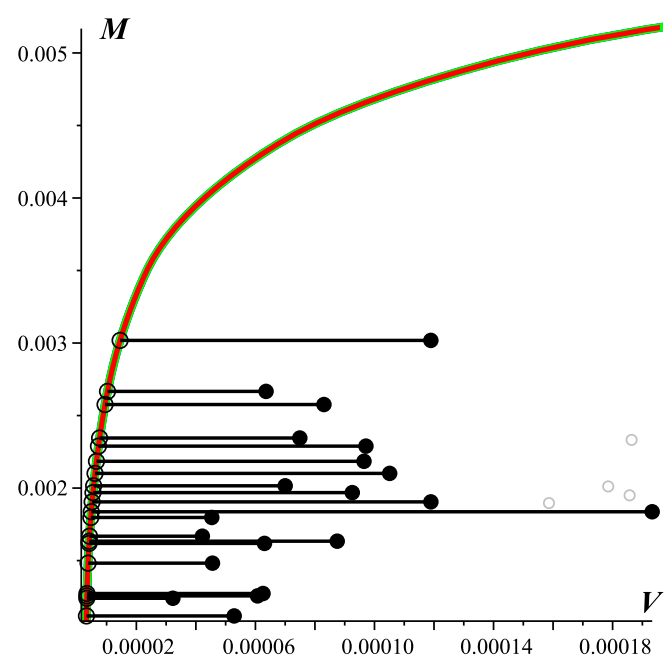

(c)

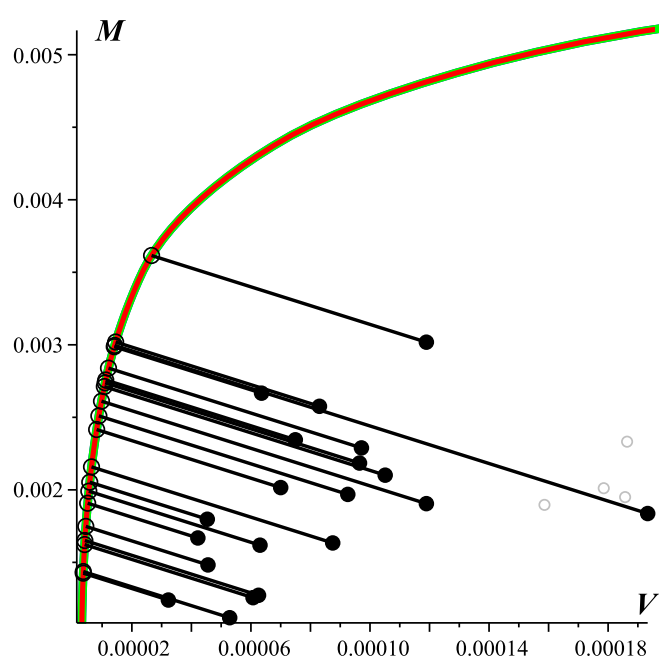

(b)

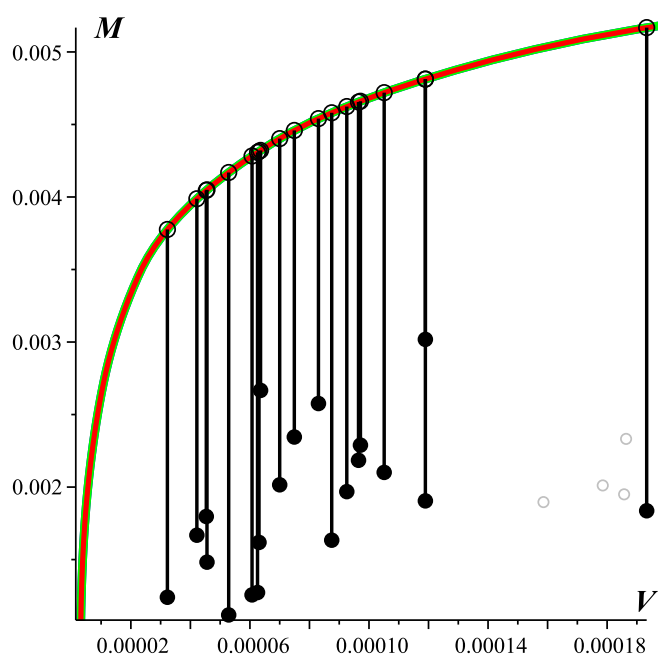

(d) 
Figure 6: Visualisation of the curves of equal efficiency according to different projection schemes

For each point of a 2 d-grid covering $\Phi(\Im)$, the efficiency measure is computed, following different projection schemes. The efficiency values obtained are scaled cyclical to a grey tone value for colouring the corresponding grid point. In this way, the curves of equal efficiency become visible. The following projection schemes are applied: (a) The PD-projection scheme; (b) The UPD-projection scheme; (c) The FD- and UFD-projection scheme in a horizontal direction; (d) The UFD- and UFDprojection scheme in a vertical direction; (e) The FD- and UFD-projection scheme in a slant direction. The original assets are visible as small green dots.

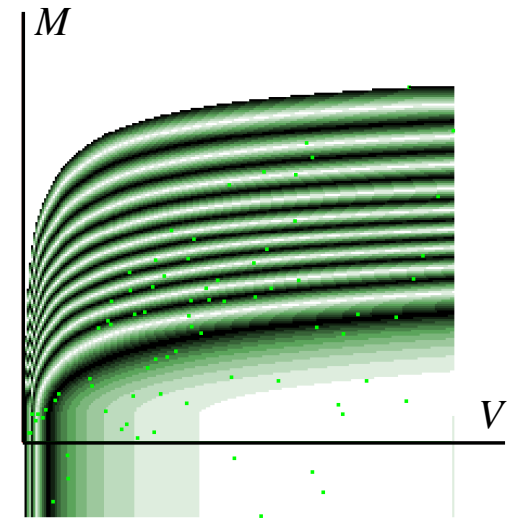

(a)

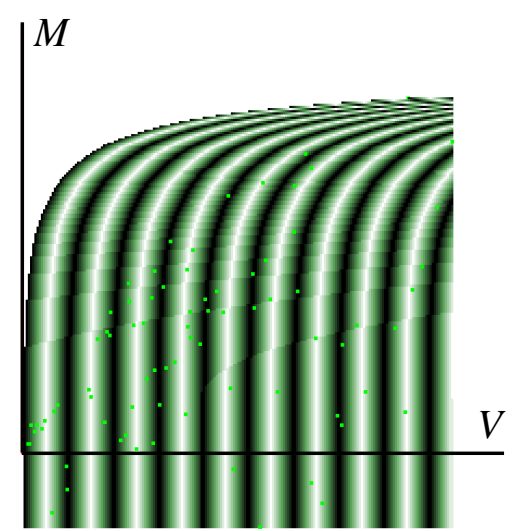

(c)

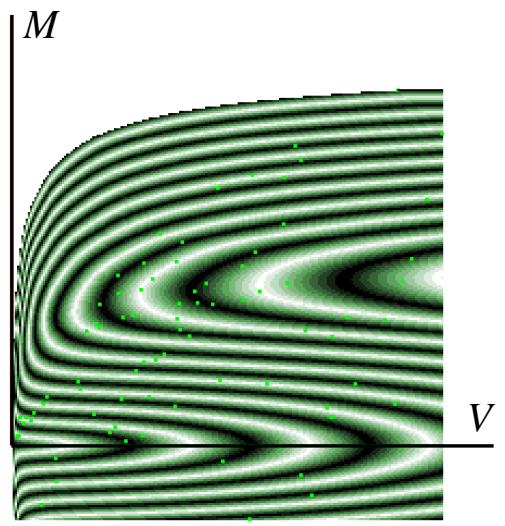

(b)

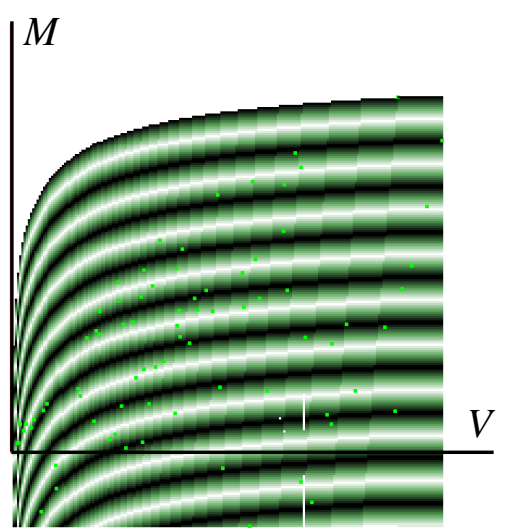

(d)

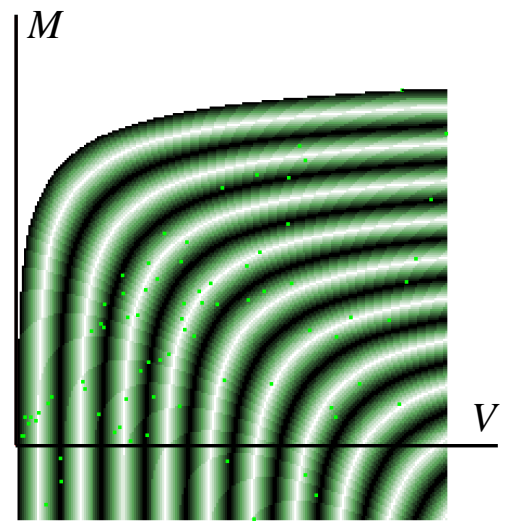

(e) 